\title{
MONOTONE DECOMPOSITIONS OF HAUSDORFF CONTINUA
}

\author{
ELDON J. VOUGHT
}

\begin{abstract}
A monotone, upper semicontinuous decomposition of a compact, Hausdorff continuum is admissible if the layers (tranches) of the irreducible subcontinua of $M$ are contained in the elements of the decomposition. It is proved that the quotient space of an admissible decomposition is hereditarily arcwise connected and that every continuum $M$ has a unique, minimal admissible decomposition \&. For hereditarily unicoherent continua $\mathbb{Q}$ is also the unique, minimal decomposition with respect to the property of having an arcwise connected quotient space. A second monotone, upper semicontinuous decomposition $\mathcal{G}$ is constructed for hereditarily unicoherent continua that is the unique minimal decomposition with respect to having a semiaposyndetic quotient space. Then $\mathscr{Q}$ refines $\mathcal{G}$ and $\mathcal{G}$ refines the unique, minimal decomposition $\mathfrak{L}$ of FitzGerald and Swingle with respect to the property of having a locally connected quotient space (for hereditarily unicoherent continua).
\end{abstract}

For a compact, Hausdorff continuum $M$, FitzGerald and Swingle [4, p. 37] have obtained a unique monotone, upper semicontinuous decomposition $\mathfrak{L}$ whose quotient space $(M, \mathfrak{L})$ is semilocally connected and which is minimal with respect to these properties. A second description of this decomposition using collections of closed subsets which separate $M$ is given by them in [4, p. 49] and by McAuley in [9, p. 2]. For a compact, metric continuum $M$, Charatonik has defined a decomposition to be admissible if it is monotone, upper semicontinuous and the layers of the irreducible subcontinua of $M$ are contained in the elements of the decomposition [2, pp. 115-116]. He then proves in the same paper that the quotient space of an admissible decomposition of a continuum $M$ is hereditarily arcwise connected and that every continuum has a unique, minimal admissible decomposition $Q$. One purpose of this paper is to extend Charatonik's results to compact, Hausdorff continua.

A second purpose pertains to the class of hereditarily unicoherent, Hausdorff continua. It is easily seen with simple examples that in general the two decompositions mentioned above are not comparable, i.e., neither refines the other. However, if the continuum $M$ is hereditarily unicoherent then $\mathbb{Q}$ refines $\mathcal{L}(\mathbb{Q} \leqq \mathfrak{L})$ since the semilocally connected quotient space $(M, \mathfrak{L})$ must then be arcwise connected. In this paper a third decomposition $\mathcal{S}$ is constructed which is the unique minimal decomposition with respect to being monotone, upper semicontinuous and having a semiaposyndetic quotient space. The construction involves a collection of closed subsets of $M$ that separate $M$ and satisfies

Received by the editors September 10, 1974 and, in revised form, January 14, 1975.

AMS (MOS) subject classifications (1970). Primary 54B15; Secondary 54F20. 
a condition used in $[4$, p. 49]. It will then be the case that $\mathscr{Q} \leqq \mathcal{G} \leqq \mathcal{L}$ where none of these refinements can be reversed.

In a compact, Hausdorff continuum an arc (sometimes called a generalized arc) is a subcontinuum with exactly two nonseparating points. If $M$ is an irreducible continuum Gordh has proved [6, pp. 648-649] that $M$ has a unique minimal monotone, upper semicontinuous decomposition $\mathscr{D}$ such that the quotient space is either degenerate or an arc. The elements of this unique decomposition are called layers and if $q$ denotes the quotient map of $M$ onto $(M, \mathcal{D})$, the layers are the sets of the form $q^{-1}(x)$ where $x \in(M, \mathscr{D})$. If $F$ is a set function then $F$ is expansive if whenever $A \subset M$ then $A \subset F(A)$ and if $A \subset B \subset M$, then $F(A) \subset F(B)$. If $A \subset M$ then $A$ is $F$-closed if $A=F(A)$. In order to generalize Charatonik's decomposition theorems to the Hausdorff setting we begin by defining the set function $P$ in the compact, Hausdorff continuum $M$. If $A \subset M$ then $P(A)$ is the union of all the layers of irreducible subcontinua of $M$ that intersect $A$.

THEOREM 1. If $M$ is a compact, Hausdorff continuum then $M$ admits a unique minimal admissible decomposition $\mathbb{Q}$.

Proof. If $A, B \subset M$ it is clear that $A \subset P(A)$ and if $A \subset B$ then $P(A)$ $\subset P(B)$. Therefore $P$ is expansive. By $[4$, p. 37, Theorem 2.5] $M$ has a unique minimal decomposition $\mathbb{Q}$ with respect to the properties of being upper semicontinuous and having $P$-closed elements. Let $\mathbb{Q}^{\prime}$ be the upper semicontinuous decomposition whose elements are the components of the elements of $Q$. If $A \in \mathbb{Q}$ and $C_{A}$ is a component of $A$ then $P\left(C_{A}\right) \subset P(A)=A$ since $A$ is $P$ closed. But $P\left(C_{A}\right)$ is clearly connected so $P\left(C_{A}\right)=C_{A}$ since $C_{A}$ is a component of $A$. Thus the elements of $\mathbb{Q}^{\prime}$ are $P$-closed and because $\mathbb{Q}$ refines every decomposition with this property we must have $\mathscr{Q}^{\prime}=\mathscr{Q}$. Therefore $\mathscr{Q}$ is monotone. If $A \in \mathbb{Q}$ and $L$ is a layer of an irreducible subcontinuum of $M$ that intersects $A$, it follows from the definition of $\bar{P}$ that $L \subset A$ since $A$ is $P$-closed. Hence $\mathcal{Q}$ is an admissible decomposition. Now if $\beta$ is any other admissible decomposition then clearly the elements of $\beta$ must be $P$-closed and so $\mathbb{Q} \leqq \beta$ since $\mathscr{Q}$ refines every upper semicontinuous decomposition into $P$-closed elements. Therefore $\mathcal{Q}$ is the decomposition as claimed in the theorem.

If $A, B, C \subset M$ then $A$ cuts $B$ from $C$ if every subcontinuum of $M$ that intersects both $B$ and $C$ also intersects $A$.

TheOREM 2 (BING). Let $M$ be a compact, Hausdorff continuum irreducible between points $a$ and $b$. If no point $x$ in $M$ cuts any other point $y$ from $\{a, b\}$ then $M$ is an arc.

Proof. The proof given by Bing [1, Theorem 6] for compact, metric continua generalizes immediately to the Hausdorff setting.

THEOREM 3. Let $M$ be a compact, Hausdorff continuum and let $\beta$ be an admissible decomposition. Then the quotient space $(M, \beta)$ is hereditarily arcwise connected.

Proof. To show $(M, \beta)$ is hereditarily arcwise connected, it is clear that it suffices to show that every nondegenerate, irreducible subcontinuum of $(M, \beta)$ is an arc. Let $N$ be an irreducible subcontinuum of $(M, \beta)$ between $a$ and $b$, 
$a \neq b$. Take $x, y \in N, x \neq y$, and let us show that $x$ does not cut $y$ from $\{a, b\}$. Then by Theorem $2, N$ will be an arc. Let $f$ be the quotient map of $M$ onto $(M, \beta)$ and consider the continuum $f^{-1}(N)$. Let $T$ be an irreducible subcontinuum of $f^{-1}(N)$ from $f^{-1}(y)$ to $f^{-1}(b)$. If $f^{-1}(x) \cap T=\varnothing$ then $x$ $\notin f(T), b, y \in f(T)$ and $x$ does not cut $y$ from $\{a, b\}$ (we are assuming here that neither $x$ nor $y$ is $a$ or $b$; if $x$ or $y$ is one of the points $a$ or $b$ the argument will be clear from what follows). Assume $f^{-1}(x) \cap T \neq \varnothing$. Because $\beta$ is admissible the layers of $T$ lie in the elements of $\left\{f^{-1}(s) \mid s \in(M, \beta)\right\}$. Also $T$ is irreducible from $f^{-1}(y)$ to $f^{-1}(b)$ and it follows that $T$ has a subcontinuum $T^{\prime}$ such that $T^{\prime} \cap f^{-1}(x) \neq \varnothing \neq T^{\prime} \cap f^{-1}(b)$ and $T^{\prime} \cap f^{-1}(y)=\varnothing$. Let $S$ be an irreducible subcontinuum of $f^{-1}(N)$ from $f^{-1}(a)$ to $f^{-1}(x)$. If $S$ $\cap f^{-1}(y)=\varnothing$ then $f(S) \cup f\left(T^{\prime}\right)$ is a subcontinuum of $N$ containing $a$ and $b$ but not $y$ contradicting the irreducibility of $N$. So $S \cap f^{-1}(y) \neq \varnothing$ and by the same reasoning as above there exists a subcontinuum $S^{\prime}$ of $S$ such that $S^{\prime} \cap f^{-1}(y) \neq \varnothing \neq S^{\prime} \cap f^{-1}(a)$ while $S^{\prime} \cap f^{-1}(x)=\varnothing$. Then $a, y$ $\in f\left(S^{\prime}\right)$ but $x \notin f\left(S^{\prime}\right)$ and $x$ does not cut $y$ from $\{a, b\}$ thus completing the proof.

Corollary. If $M$ is a compact, Hausdorff continuum and $\mathbb{Q}$ is the unique minimal admissible decomposition of $M$, then $(M, \mathbb{Q})$ is hereditarily arcwise connected.

The structure of the elements of the unique minimal admissible decomposition $(M, \mathscr{Q})$ of $M$ is described in [4, $\S \$ 4$ and 7] by replacing the set function $N$ by the set function $P$ used here.

A continuum $M$ is hereditarily unicoherent if the intersection of any two intersecting subcontinua is connected. The following two notions of aposyndesis and semilocal connectedness are equivalent for compact, Hausdorff continua [7, pp. 546-547]. We say $M$ is semilocally connected if given $x \in M$ and an open set $U$ containing $x$, there exists an open set $V$ such that $x \in V \subset U$ and $M-V$ consists of a finite number of components. The continuum $M$ is aposyndetic if given distinct points $x, y$ of $M$ there exist open sets $U_{x}, U_{y}$ and continua $H_{x}, H_{y}$ such that $x \in U_{x} \subset H_{x} \subset M-\{y\}$ and $y$ $\in U_{y} \subset H_{y} \subset M-\{x\}$. If one of these sequences of inclusions (but not necessarily both) can always be obtained for distinct points $x, y \in M$, then $M$ is semiaposyndetic. If $B \subset M$ let $T(B)=B \cup\{z \in M \mid$ there does not exist an open set $U$ and continuum $H$ such that $z \in U \subset H \subset M-B\}$ and $K(B)$ $=B \cup\{z \in M \mid$ there does not exist an open set $U$ and continuum $H$ such that $B \subset U \subset H \subset M-\{z\}\}$. These two set functions $T$ and $K$ are due to $\mathrm{F}$. B. Jones [8, pp. 404-405]. Now let $Z$ be the set function defined by $Z(B)=K(B) \cap T(B)$ where $B \subset M$. We turn our attention now to hereditarily unicoherent continua and use $Z$ to describe a decomposition $\mathcal{G}$ that "fits" between $\mathscr{Q}$ and $\mathscr{C}$.

THEOREM 4. Let $M$ be a compact Hausdorff continuum. There exists a unique minimal upper semicontinuous decomposition $\mathcal{G}$ of $M$ into Z-closed elements.

Proof. Clearly for all subsets $A, B$ of $M, A \subset Z(A)$ and if $A$ $\subset B$ then $Z(A) \subset Z(B)$. So the set function $Z$ is expansive and the required decomposition exists by [4, Theorem 2.5]. 
Lemma 1. Let the compact, Hausdorff continuum $M$ be hereditarily unicoherent and let $A \subset M$. If $A$ is connected then $Z(A)$ is a continuum.

Proof. It is known that $T(A)$ is a continuum [4, p. 35]. In general $K(A)$ is not connected but because $M$ is hereditarily unicoherent it follows easily that $K(A)$ is a continuum. Thus $Z(A)=T(A) \cap K(A)$ is a continuum.

THEOREM 5. Let $M$ be a hereditarily unicoherent compact Hausdorff continuum and let $\mathcal{G}$ denote the decomposition of Theorem 4 . Then (i) $\mathcal{G}$ is upper semicontinuous, (ii) $\mathcal{G}$ is monotone, (iii) $(M, \mathcal{G})$ is semiaposyndetic, and (iv) $\mathcal{G}$ refines every decomposition satisfying (i), (ii), and (iii).

Proof. Let $\mathcal{G}^{\prime}$ be the upper semicontinuous decomposition of $M$ whose elements are components of members of $\mathcal{G}$. Using Lemma 1 and the argument given in Theorem 1 the elements of $\mathcal{G}^{\prime}$ are $Z$-closed. Since $\mathcal{G}$ refines every such decomposition it must be that $\mathcal{G}=\mathcal{G}^{\prime}$ and $\mathcal{G}$ is thus monotone. Now let $x, y \in(M, \mathcal{G}), x \neq y$. Then $f^{-1}(x)$ and $f^{-1}(y)$ are connected and Z-closed where $f$ is the quotient map of $M$ onto $(M, \mathcal{G})$. Because $M$ is hereditarily unicoherent either $K\left(f^{-1}(x)\right) \cap f^{-1}(y)=\varnothing$ or $T\left(f^{-1}(x)\right) \cap f^{-1}(y)=\varnothing$. Otherwise

$$
\left(K\left(f^{-1}(x)\right) \cup f^{-1}(y)\right) \cap\left(T\left(f^{-1}(x)\right) \cup f^{-1}(y)\right)=f^{-1}(x) \cup f^{-1}(y)
$$

which is not connected. If $K\left(f^{-1}(x)\right) \cap f^{-1}(y)=\varnothing$ then because $M$ is hereditarily unicoherent and $f^{-1}(y)$ is compact, there exists an open set $W$ and continuum $H$ such that $f^{-1}(x) \subset W \subset H$ and $H \cap f^{-1}(y)=\varnothing$. If $T\left(f^{-1}(x)\right) \cap f^{-1}(y)=\varnothing$, by the compactness of $f^{-1}(y)$ there exists $W$ and $H$ as before such that $f^{-1}(y) \subset W \subset H$ and $H \cap f^{-1}(x)=\varnothing$. Because $\mathcal{G}$ is upper semicontinuous it follows that either $x \in f(H)^{0} \subset f(H) \subset(M, \mathcal{G})$ $-\{y\}$ or $y \in f(H)^{0} \subset f(H) \subset(M, \mathcal{G})-\{x\}$. Hence $(M, \mathcal{G})$ is semiaposyndetic.

Finally let $\mathcal{G}^{\prime}$ be any monotone decomposition of $M$ such that $\left(M, \mathcal{G}^{\prime}\right)$ is semiaposyndetic and we will show that $\mathcal{G} \leqq \mathcal{G}^{\prime}$. From the semiaposyndesis of $\left(M, \mathcal{G}^{\prime}\right)$ it follows that $\left(M, \mathcal{G}^{\prime}\right)$ is a Hausdorff space and this implies that $\mathcal{G}^{\prime}$ is upper semicontinuous. Take $G \in \mathcal{G}^{\prime}, x \in M-G$ and let $a=f(G), b=f(x)$ where $f$ is the quotient map of $M$ onto $\left(M, \mathcal{G}^{\prime}\right)$. Because $\left(M, \mathcal{G}^{\prime}\right)$ is semiaposyndetic we can assume that there exist an open set $W$ and continuum $H$ of $\left(M, \mathcal{G}^{\prime}\right)$ such that $a \in W \subset H \subset\left(M, \mathcal{G}^{\prime}\right)-\{b\}$. Then $G \subset f^{-1}(W)$ $\subset f^{-1}(H) \subset M-\{x\}$. Hence $x \notin K(G)$ and thus $x \notin Z(G)$ which shows that the elements of $\mathcal{G}^{\prime}$ are $Z$-closed. Since $\mathcal{G}^{\prime}$ is upper semicontinuous with $Z$ closed elements and $\mathcal{G}$ is the unique minimal such decomposition, it must be that $\mathcal{G} \leqq \mathcal{G}^{\prime}$.

Now let us describe the unique minimal decomposition of Theorem 4 by an internal construction. Let $K$ be a collection of closed subsets of $M$ each of which separates $M$ (called closed separators) with the following property:

If $k \in K$ and $M-k=A_{1} \cup A_{2}$, a separation, then if

(*) $\quad a_{1} \in A_{1}, a_{2} \in A_{2}, b \in k$, there exist $k^{\prime} \in K$ and for either $i=1, j=2$ or $j=1, i=2$ an open set $W$ and continuum $H$ such that $a_{i} \in W \subset H$ and $k^{\prime}$ separates $a_{j}$ from $H \cup\{b\}$. 
Let $\tilde{K}$ be the union of all the collections of closed separators satisfying (*). Then $\tilde{K}$ is itself a collection of closed separators satisfying (*) and is clearly the unique maximal such collection. Given $x \in M$ denote by $S_{x}$ the set of all points $y$ of $M$ such that there does not exist $k \in \tilde{K}$ which separates $x$ from $y$. By [4, Lemma 8.1] $\delta=\left\{S_{x} \mid x \in M\right\}$ is an upper semicontinuous decomposition of $M$.

THEOREM 6. If $\mathcal{G}$ is the unique minimal decomposition of Theorem 4 with respect to being upper semicontinuous with Z-closed elements for the compact, Hausdorff, hereditarily unicoherent continuum $M$, then $\delta=\mathcal{G}$.

Proof. Let $f$ be the quotient map of $M$ onto $(M, \mathcal{S})$ and let $K=\left\{f^{-1}(k) \mid k\right.$ is a closed separator of $(M, \mathcal{G})\}$. Now $K$ is a collection of closed separators of $M$. Suppose $M-f^{-1}(k)=A \cup B$, a separation, and $a \in A, b \in B, c$ $\in f^{-1}(k)$. Because $f$ is monotone $k$ separates $f(a)$ from $f(b)$ in $(M, \mathcal{G})$ and because $(M, \mathcal{G})$ is semiaposyndetic there exist an open set $W$ and continuum $H$ of $(M, \mathcal{G})$ such that $f(a) \in W \subset H \subset(M, \mathcal{S})-\{f(b)\}$. Let $k^{\prime}$ be a closed set that separates $f(b)$ from $H \cup\{f(c)\}$. Then $f^{-1}\left(k^{\prime}\right)$ separates $b$ from $f^{-1}(H)$ $\cup\{c\}$ in $M$. Hence $K$ has property $(*)$. This implies that $\delta \leqq \mathcal{G}$ for suppose that $S_{x} \in \mathcal{S}$ and $G \cap S_{x} \neq \varnothing \neq G^{\prime} \cap S_{x}$ where $G, G^{\prime} \in \mathcal{G}$ and $G \neq G^{\prime}$. Let $k$ be a closed subset of $(M, \mathcal{G})$ that separates $f(G)$ from $f\left(G^{\prime}\right)$. It follows that $f^{-1}(k)$ separates $G$ from $G^{\prime}$ so $f^{-1}(k)$ separates two points of $S_{x}$. This contradicts the definition of $S_{x}$ since $f^{-1}(k)$ belongs to $K$ and therefore to $\tilde{K}$.

Denote by $\delta_{c}$ the set of all components of members of $\delta$. Clearly $\delta_{c} \leqq \delta$ $\leqq \mathcal{G}$ and the proof will be complete by showing that $\mathcal{S} \leqq \delta_{c}$, which implies that $\delta=\mathcal{G}$. To prove this we will show that $\delta_{c}$ is upper semicontinuous with $Z$-closed elements. Because $\mathcal{G}$ is minimal with respect to these properties it will then follow that $\mathcal{G} \leqq \delta_{c}$. Clearly $\delta_{c}$ is upper semicontinuous since $\delta$ is. Take $S \in \mathcal{S}, x \in M-S$ and let $C_{s}$ be a component of $S$. By the definition of $\tilde{K}$, for every $y \in C_{s}$ there exist $k^{\prime} \in \tilde{K}$ and a continuum $H$ such that either (1) $y \in H^{0} \subset H \subset M-\{x\}$ and $k^{\prime}$ separates $H$ from $x$ or (2) $x \in H^{0} \subset H$ $\subset M-\{y\}$ and $k^{\prime}$ separates $H$ from $y$. Suppose for some $y \in C_{s}$ that (2) is true. Then $H \cap C_{s}=\varnothing$ for otherwise $k^{\prime}$ would separate two points of $S$. Thus $x \notin T\left(C_{s}\right)$ so $x \notin Z\left(C_{s}\right)$. Now assume that for every $y \in C_{s}$ that (1) is true. By compactness there exist an open set $W$ and continuum $Q$ such that $C_{s} \subset W \subset Q \subset M-\{x\}$. Therefore $x \notin K\left(C_{s}\right)$ and so in this case also $x \notin Z\left(C_{s}\right)$. Hence $Z\left(C_{s}\right) \subset S$ and since $Z\left(C_{s}\right)$ is connected by Lemma 1 and $C_{s}$ is a component of $S, Z\left(C_{s}\right)=C_{s}$. This completes the proof.

THEOREM 7. If $M$ is a hereditarily unicoherent compact Hausdorff continuum, then $\mathcal{Q} \leqq \mathcal{G} \leqq \mathcal{L}$.

Proof. For a hereditarily unicoherent continuum $M$, Charatonik has shown [3, Theorem 5] that if $M$ has a monotone, upper semicontinuous decomposition with an arcwise connected quotient space then the decomposition is admissible. His proof, although given for metric continua, extends easily to the Hausdorff setting. Thus for hereditarily unicoherent continua the unique minimal admissible decomposition $\mathbb{Q}$ of Theorem 1 can alternately be described as the unique, minimal monotone, upper semicontinuous decomposition whose quotient space is hereditarily arcwise connected. In Theorem 5 the 
decomposition $\mathcal{G}$ is shown to be unique and minimal with respect to the properties of being monotone, upper semicontinuous and having a semiaposyndetic quotient space. But Gordh [5, Theorem 2.3] has proved that a semiaposyndetic, hereditarily unicoherent continuum is arcwise connected. So it follows from this that $\mathscr{Q} \leqq \mathcal{G}$. Now recall from the introduction that $\mathcal{L}$ is the unique, minimal, upper semicontinuous decomposition of a continuum such that the quotient space is semilocally connected. But a hereditarily unicoherent, semilocally connected continuum is locally connected, so for hereditarily unicoherent continua, $\mathcal{L}$ can be described as unique and minimal with respect to the properties of being monotone, upper semicontinuous and having a locally connected quotient space. Hence $\mathfrak{Q} \leqq \mathcal{G} \leqq \mathcal{L}$.

It is easily seen with simple examples that these refinements cannot be reversed. Also by forming a continuum that includes a $\sin (1 / x)$ curve and its limit bar, a simple fan, the union of two simple fans in "opposite" directions with a common limit interval, it is instructive to see how the successive application of these three decompositions to this continuum continually "improves" the quotient space.

\section{REFERENCES}

1. R. H. Bing, Some characterizations of arcs and simple closed curves, Amer. J. Math. 70 (1948), 497-506. MR 10, 55.

2. J. J. Charatonik, On decompositions of continua, Fund. Math. 79 (1973), 113-130. MR 47 \#9567.

3. - On decompositions of $\lambda$-dendroids, Fund. Math. 67 (1970), 15-30. MR 41 \#6169.

4. R. W. FitzGerald and P. M. Swingle, Core decompositions of continua, Fund. Math. 61 (1967),33-50. MR 36 \#7110.

5. G. R. Gordh, Jr., Concerning closed quasi-orders on hereditarily unicoherent continua, Fund. Math. 78 (1973), 61-73. MR 48 \#1196.

6. - Monotone decompositions of irreducible Hausdorff continua, Pacific J. Math. 36 (1971), 647-658. MR 43 \#6882.

7. F. B. Jones, Aposyndetic continua and certain boundary problems, Amer. J. Math. 63 (1941), 545-553. MR 3, 59.

8. - Concerning non-aposyndetic continua, Amer. J. Math. 70 (1948), 403-413. MR 9, 606.

9. L. F. McAuley, An atomic decomposition of continua into aposyndetic continua, Trans. Amer. Math. Soc. 88 (1958), 1-11. MR 23 \# A1353.

Department of Mathematics, California State University, Chico, California 95926 